\title{
Dynamic variational principles and extended coupled cluster techniques
}

\author{
Document Version \\ Accepted author manuscript
}

Link to publication record in Manchester Research Explorer

\section{Citation for published version (APA):}

Bishop, RF., Arponen, J., \& Pajanne, E. (1989). Dynamic variational principles and extended coupled cluster techniques. In D. Mukherjee (Ed.), Aspects of Many-Body Effects in Molecules and Extended Systems (pp. 79100). (Series on Lecture Notes in Chemistry; Vol. 50). Springer Nature.

http://personalpages.manchester.ac.uk/staff/raymond.bishop/RFB_papers/[067] LectNotesChem_50(1989)79

Published in:

Aspects of Many-Body Effects in Molecules and Extended Systems

\section{Citing this paper}

Please note that where the full-text provided on Manchester Research Explorer is the Author Accepted Manuscript or Proof version this may differ from the final Published version. If citing, it is advised that you check and use the publisher's definitive version.

\section{General rights}

Copyright and moral rights for the publications made accessible in the Research Explorer are retained by the authors and/or other copyright owners and it is a condition of accessing publications that users recognise and abide by the legal requirements associated with these rights.

\section{Takedown policy}

If you believe that this document breaches copyright please refer to the University of Manchester's Takedown Procedures [http://man.ac.uk/04Y6Bo] or contact uml.scholarlycommunications@manchester.ac.uk providing relevant details, so we can investigate your claim.

\section{OPEN ACCESS}




\section{Lecture Notes in Chemistry}

Edited by G. Berthier M.J.S. Dewar H. Fischer K. Fukui G.G. Hall J. Hinze H.H. Jaffé J. Jortner W. Kutzelnigg K. Ruedenberg J. Tomasi

\section{0}

D. Mukherjee (Ed.)

\section{Aspects of Many-Body Effects in Molecules and Extended Systems}

Proceedings of the Workshop-Cum-Symposium Held in Calcutta, February 1-10, 1988

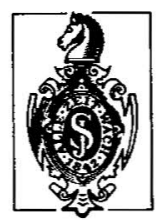

Springer-Verlag

Berlin Heidelberg New York London Paris Tokyo 
Editor

D. Mukherjee

Department of Physical Chemistry

Indian Association for the Cultivation of Science

Calcutta 700-032, India

ISBN 3-540-50765-5 Springer-Verlag Berlin Heidelberg New York

ISBN 0-387-50765-5 Springer-Verlag New York Berlin Heidelberg

Library of Congress Cataloging-in-Publication Data. Aspects of many-body effects in molecules and extended systems: proceedings of the workshop-cum-symposium, held in Calcutta, February $1-10,1988$ / edited by D. Mukherjee. p. cm.--(Lecture notes in chemistry; 50) Includes index. ISBN 0-387-50765-5

1. Molecular structure. 2. Many-body problem. I. Mukherjee, D. (Debashis), 1946-. II. Series. QD461.A79 1989 541.2'2-dc 19 89-4164 CIP

This work is subject to copyright. All rights are reserved, whether the whole or part of the material is concerned, specifically the rights of translation, reprinting, re-use of illustrations, recitation, broadcasting, reproduction on microfilms or in other ways, and storage in data banks. Duplication of this publication or parts thereof is only permitted under the provisions of the German Copyright Law of September 9,1965, in its version of June 24, 1985, and a copyright fee must always be paid. Violations fall under the prosecution act of the German Copyright Law.

(C) Springer-Verlag Berlin Heidelberg 1989

Printed in Germany

Printing and binding: Druckhaus Beltz, Hemsbach/Bergstr. 
DYNAMIC VARIATIONAL PRIACIPLES AND

EXTENDED COUPLED CLUSTER TECHNIQUES

\author{
R.F. Bishop \\ Department of Mathematics \\ University of Manchester Institute of Science and Technology \\ P.0. Box 88, Manchester M60 1QD, England
}

and

J. Arponen and E. Pajanne

Research Institute for Theoretical Physics

Siltavuorenpenger 20C, 00170 Helsinki, Finland

Abstract: We present a description of the so-called extended coupled cluster method (ECCM), and show how it provides a rather powerful and general treatment for the quanturm-mechanical many-body problem. Since this method has to date not yet been applied to systems of interest in quantum chemistry, we compare and contrast the method with both the configuration-interaction (CI) and (normal) coupled cluster (CC) techniques which have been so successfulity applied to a wide variety of many-body systems, including atoms and molecules. We indicate the potential advantages of the ECCM over these other methods, and show how in some very real sense it completes the process begun but not completed by the normal CC method in attempting to overcome the (size-extensivity) problems associated with the CI method. The ECCM provides, in principle, an exact parametrisation of the many-body Hilbert space in terms of a complete set of linked-cluster amplitudes, all of which obey the cluster property. By contrast, none of the corresponding CI amplitudes, and only half of the normal CC amplitudes, obey this property. This has important consequences if one is interested in arbitrary expectation values or geometric (topological) properties. Both static and dynamic properties are describable, and we sketch the ECCM description of both ground and excited states.

\title{
1. INTRODUCTION
}

Coupled cluster (CC) techniques [1-9] have become rather well known to quantum chemists in view of the many extremely successful and accurate calculations that have by now been performed on a wide variety of atoms and molecules, for both closed-shell and open-shell systems. Indeed, several of the other articles in the present volume review the present state of the art of these calculations, and give extensive references to the literature that we do not attempt to reproduce here. This large corpus of work in quantum chemistry (and see, e.g., Refs. [3,6,10-14] and other articles in this volume) is replicated by similar successes of the CC techniques in such other diverse areas as nuclear physics [4,7] (-- both finite nuclei and infinite nuclear matter), the homogeneous electron gas [5,15-17], systems of anharmonic osci] 
[18], and systems in relativistic quantum field theory [19].

All of the above applications stem from the original work by Coester and Kümmel $[1,2]$ on what they called the $\exp (S)$ method, and which was later to become known as the coupled cluster method (CCM). In particular we now wish to call it the normal coupled cluster method (NCCM) to distinguish it from its much more recent generalisation -- the so-called extended coupled cluster method (ECCM) [20-22], which is our main concern here. A1l of the quantum chemistry calculations to date, which are described elsewhere in this volume, are NCCM calculations in our parlance. One of our primary aims here is to describe the additional virtues and features that the ECCM possesses, which we believe may make it a very valuable additional tool for further progress in the field.

We stress how in one light the ECCM may be viewed as a particular scheme for summing the complete class of Goldstone diagrams of time-independent perturbation theory. We shall particularly show here how the way in which it groups the various terms (or diagrams) together, differs from the corresponding configuration-interaction (CI) [23] and NCCM schemes. The ECCM naturally groups these diagrams in terms of generalised tree diagram structures that have a generalised time-ordering (g.t.0.) property both forwards and backwards in time. By contrast, the g.t.o. property only holds in the backward time-direction for the corresponding NCCM tree diagrams.

We also point out here that at each natural level of approximation, the average value $\bar{A}$ of some arbitrary operator $A$, is obtained within the ECCM from just the same set of Goldstone diagrams as for the energy -- by replacing each Hamiltonian vertex in the diagram in turn by the operator $A$. We further show how such average values $\bar{A}$ may be expressed entirely in terms of the basic linked-cluster amplitudes $\left\{\sigma_{n}, \tilde{\sigma}_{n}\right\}$, which themselves completely parametrise the many-body system within the ECCM. Our dynamic variational approach enables us to set up a formally exact set of coupled nonlinear equations of motion for these basic amplitudes. We show how these may be regarded as providing a mapping of the original quantum many-body theory into a generalised classical Hamiltonian mechanics for the basic amplitudes $\left\{\sigma_{n}, \tilde{\sigma}_{n}\right\}$, which are thereby seen to play the role of a complete set of generalised quasi-local mean classical fields in the many-body configuration space. Finally, we make connection with the excited states of the many-body system, and show explicitly how the collective eigermodes may also be obtained by linearising the previous equations of motion

for the amplitudes $\left\{\sigma_{n}, \tilde{\sigma}_{n}\right\}$ to allow small oscillations around the ground-state equilibrium.

\section{CONFIGURATION SPACE CONCEPTS}

The ECCM formalism is extremely general, and may be applied to a11 quantummechanical problems for which a Schrödinger dynamics is defined. In order to keep the discussion as general as possible, we first define our operator algebra rather broadly. However, when we need to be definite we shall, for purposes of this paper, 
restrict ourselves mostly to systems of bosons. The necessary extensions to systems of fermions or to spin-algebraic systems, etc., are mostly straightforward.

We shall see that the fundamental building-blocks of the ECCM are subsystem amplitudes or linked-configuration excitation amplitudes. We begin by introducing a shorthand notation for the operators and amplitudes in a general many-body Hilbert space, and a method to compound the resulting configuration-space indices. We postulate only the existence of some initial or model state $|\Phi\rangle$ in the many-body Hilbert space $H$. We further assume that the algebra of all operators in $H$ is spanned by the two subalgebras of creation and destruction operators with respect to the state $|\Phi\rangle$. Our basic postulate is that these two Abelian subalgebras and the state $|\Phi\rangle$ are cyclic in the sense that all of the ket states in $H$ can be constructed from linear combinations of the states reached by operating on $|\Phi\rangle$ with the elements of the creation-operator subalgebra; and equivalently for the bra states with respect to $<\Phi \mid$.

We thus denote an arbitrary product of single-particle creation operators by the symbol $C_{i}^{\dagger}$, where the index $i$ is a compact notation for a configuration (or collection) of single-particle labels.

We assume that these operators are orthonormalised,

$$
\left\langle\Phi\left|C_{i} C_{j}^{\dagger}\right| \Phi\right\rangle=\delta(i, j),
$$

where $\delta(i, j)$ is a suitable Kronecker symbol. The elements of the set $\left\{C_{i}^{\dagger}\right\}$ all commute with each other. The identity operator I has the resolution,

$$
\begin{aligned}
I & =|\Phi><\Phi|+\sum_{i}^{\prime} C_{i}^{\dagger}|\Phi><\Phi| C_{i} \\
& =\sum_{i} C_{i}^{\dagger}|\Phi><\Phi| C_{i},
\end{aligned}
$$

where the prime on the summation restricts us to those $c_{i}^{\dagger}$ which create at least one particle $\{i \neq 0\}$. Conversely, an unprimed sum on configuration-space indices includes the term $i=0$ with $C_{0}^{\dagger} \equiv I \equiv C_{0}$. For example, if $|\Phi\rangle$ is taken to be the vacuum state $\mid 0>$, and the single-particle creation operators (or field operators) $\underset{\mathrm{x}}{\mathrm{a}_{\overrightarrow{\mathrm{C}}}^{+}}$create a boson at space-point $\vec{x} \equiv\left(\mathrm{x}^{1}, \mathrm{x}^{2}, \mathrm{x}^{3}\right)$, then

$$
c_{i}^{\dagger} \rightarrow(n !)^{-\frac{1}{2}} \stackrel{n}{\prod_{m=1}} \underset{\vec{x}_{m}}{a^{\dagger}},
$$

and the configuration-space index $i \rightarrow\left(\vec{x}_{1}, \cdots, \vec{x}_{n}\right)$.

The assumption that $|\Phi\rangle$ is a cyclic vector guarantees that arbitrary ket states $\mid K>$ and bra states $<B \mid$ have a decomposition, 


$$
\begin{array}{ll}
|K\rangle=\sum_{i} k_{i} C_{i}^{\dagger}|\Phi\rangle ; & k_{i}=\left\langle\Phi\left|C_{i}\right| K\right\rangle, \\
\langle B|=\sum_{i} b_{i}\langle\Phi| C_{i} ; & b_{i}=\left\langle B\left|C_{j}^{\dagger}\right| \Phi\right\rangle,
\end{array}
$$

We further define "addition" and "subtraction" of the configurations as follows:

$$
\begin{aligned}
& C_{i+j}^{\dagger} \equiv C_{j}^{\dagger} C_{j}^{\dagger} ; \quad C_{i+j} \equiv C_{i} C_{j} ; \\
& \left.C_{i-j}^{\dagger}\left|\Phi>\equiv C_{j} C_{i}^{\dagger}\right| \Phi\right\rangle \quad ; \quad\langle\Phi| C_{i-j} \equiv\langle\Phi| C_{i} C_{j}^{\dagger}
\end{aligned}
$$

The operator $c_{i-j}^{+}$is thus defined to be the creation part of the full contraction of the product $C_{j} C_{j}^{\dagger}$. It is therefore zero unless the index set $j$ is a proper subset of the index set $i$. The compounded expansion coefficients are similary defined as:

$$
\begin{aligned}
& k_{j+j} \equiv\left\langle\Phi\left|C_{j+j}^{\dagger}\right| K\right\rangle=\sum_{n} k_{n}\left\langle\Phi\left|C_{i} C_{j} C_{n}^{\dagger}\right| K\right\rangle ; \\
& k_{j-j} \equiv\left\langle\Phi\left|C_{i-j}^{\dagger}\right| K\right\rangle=\sum_{n} k_{n}\left\langle\Phi\left|C_{j} C_{j}^{\dagger} C_{n}^{\dagger}\right| K\right\rangle,
\end{aligned}
$$

The reader should be warned that these laws of "addition" and "subtraction" of configurations are neither associative nor commutative in general, and care must be taken accordingly.

We note finally that it is often useful to arrange operators products into normal order. We have shown elsewhere [21] that a very useful form of the Wick theorem for this purpose may be expressed in the above notation as,

$$
c_{j} C_{i}^{\dagger}=\sum_{n} Z_{n}(i, j) C_{i-n}^{\dagger} C_{j-n}
$$

where $Z_{n}(i, j)$ is a numerical coefficient which depends on the system in question. For the vacuum Bose system used as example above, the factor $Z_{n}(i, j)=1$.

\section{DYNAMIC VARIATION PRINCIPLE AND GENERALISED TREE GIAGRAMS}

As the starting-point from which to generate the fundamental equations of motion, we require the "action" fronctional,

$$
A=A\left[\Psi, \Psi^{\prime}\right] \equiv \int d t\left\langle\Psi^{\prime}(t)|(i \partial / \partial t-H)| \Psi(t)\right\rangle,
$$

where $H$ is the many-body Hamiltonian, to be stationary against small (independent) variations in the wavefunctions $|\Psi\rangle$ and $\left\langle\psi^{\mathrm{I}}\right|$. Since we do not restrict ourselves to later parametrisations that explicitly preserve the hermitian adjoint relation between the states $|\Psi(t)\rangle$ and $\left\langle\Psi^{\prime}(t)\right|$, we remind ourselves of this fact by the prime notation on the bra state. The dynamic variational principle thus generates 
the time-dependent Schrödinger equations

$$
\begin{aligned}
& \delta \mathcal{A} / \delta \Psi^{\prime}=0 \Longrightarrow i \frac{\partial}{\partial t}|\Psi\rangle=H|\Psi\rangle ; \\
& \delta \mathcal{A} / \delta \Psi=0 \Longrightarrow-i \frac{\partial}{\partial t}\left\langle\Psi^{\prime}\right|=\left\langle\Psi^{\prime}\right| H .
\end{aligned}
$$

We now show how different exact parametrisations of the states $|\Psi\rangle$ and $\left\langle\Psi^{1}\right|$ lead to different exact reformulations of the underlying quantum many-body theory, and also, at the stationary equilibrium point, to different purely algebraic ways to generate the diagrams of time-independent perturbation theory in terns of generalised tree diagram structures. He compare and contrast below the three distinct such representations provided by (i) the configuration-interaction (CI) method, (ii) the normal coupled cluster method (NCCM), and (iii) the extended coupled cluster method (ECCM).

\subsection{The CI Representation}

The states $|\Psi\rangle$ and $\left\langle\Psi^{\prime}\right|$ are parametrised in the CI representation as,

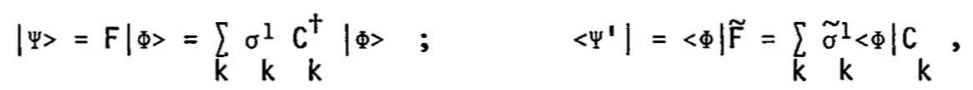

where the superscripts 1,2 and 3 on the c-number configuration-space amplitudes $\sigma_{k}$ and $\tilde{\sigma}_{k}$ will henceforth be used to distinguish respectively the three cases considered, namely: (1) the CI, (2) the NCCM, and (3) the ECCM representations. The action functional of $\mathrm{Eq}$. (7) becomes,

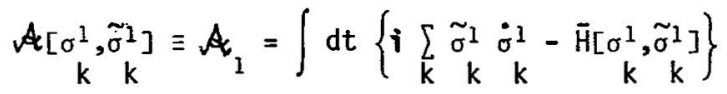

$$
\begin{aligned}
& =\int \mathrm{dt}\left\{-i \sum_{k}^{\underset{k k}{\dot{\sigma}^{1} \sigma^{1}}-\bar{H}\left[\sigma_{k}^{1}, \tilde{\sigma}_{k}^{1}\right]}\right\}
\end{aligned}
$$

where $\bar{H} \equiv\left\langle\Psi^{\mathrm{I}}|H| \Psi\right\rangle$ is given by

$$
\begin{aligned}
& \bar{H}_{1} \equiv \overline{\mathrm{H}}\left[\begin{array}{c}
\sigma^{1}, \\
\left.\tilde{\sigma}^{1}\right]
\end{array}\right] \equiv\left\langle\psi^{\prime}|H| \psi\right\rangle=\sum_{k, l}\langle k|H| \ell\rangle \underset{k \ell}{\tilde{\sigma}^{1} \sigma^{1}}, \\
& \langle k|H| \ell\rangle \equiv\left\langle\Phi\left|\mathrm{C}_{k} \mathrm{HC}_{\ell}^{\dagger}\right| \Phi\right\rangle .
\end{aligned}
$$

The requirement that $A_{1}$ be stationary with respect to arbitrary small changes in

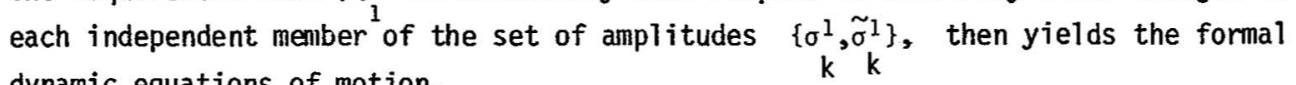
dynarnic equations of motion,

$$
i \dot{\sigma}_{k}^{1}=\delta H_{1} / \delta \tilde{\sigma}_{k}^{1} \quad ; \quad \dot{\tilde{\sigma}}_{k}^{1}=-\delta \bar{H}_{I} / \delta \sigma_{k}^{1} \quad .
$$


The ground state is given by the (stationary in time) equilibrium point,

$$
\delta \overline{\mathrm{H}}_{1} / \delta \tilde{\sigma}_{\mathrm{l}}^{1}=0=\delta \overline{\mathrm{H}}_{1} / \delta \sigma^{1} \mathrm{k} .
$$

If the Hamiltonian can be split into a sum of a one-body operator (kinetic energy) part $T$ and an interaction part $V, H=T+V$, and furthermore if the configuration space indices have been so chosen that the states $C_{k}^{\dagger}|\Phi\rangle$ are eigenstates of $T$, then it is easy to show that,

$$
\left\langle\Psi^{1}|T| \psi\right\rangle \equiv \underset{k}{\left[\sigma_{k}^{1}, \tilde{\sigma}^{1}\right] \equiv \bar{T}_{1}}=\sum_{k} \underset{k k k}{e} \tilde{\sigma}^{1} \sigma^{1}+\text { const. }
$$

where $e_{k}$ is the extra kinetic energy of the state $C_{k}^{\dagger} \mid \Phi>$ with respect to the model state $\mid \Phi>$. The static equations (13) may then be rewritten as,

$$
\underset{k}{\sigma^{1}}=-\left(e_{k}\right)^{-1} \delta \bar{V}_{1} / \delta \tilde{\sigma}_{k}^{1} ; \quad \tilde{\sigma}_{k}^{1}=-\left(e_{k}\right)^{-1} \delta \bar{V}_{1} / \delta \sigma_{k}^{1},
$$

in which form the factors $e_{k}$ now appear in the familiar guise of energy denominators. Equations (15) are precisely the usual Dyson equations for the amplitudes $\left\{\sigma^{1}, \tilde{\sigma}^{2}\right\}$ which now completely characterise the CI theory. Their iterative solution leads ${ }^{k}$ to a set of terms which can be put into one-to-one correspondence with classes of Goldstone diagrams.

Al though the resulting CI equations are conceptually simple, the method suffers from a we11-known very serious defect when applied to many-body systems. In essence, the diagrams for both sets of amplitudes $\left\{\sigma^{1}\right\}$ and $\left\{\tilde{\sigma}^{1}\right\}$ contain disconnected or unzinked pieces. A consequence is that both ${ }^{k}$ sets of amflitudes are strongly nonlocal in the sense of not obeying the cluster property of going to zero when any subset of the particles described by the corresponding configuration space index $k$ becomes far removed from the remainder. While the CI parametrisation is in principle complete and exact, in practical applications truncations are necessary. The disconnected (unlinked) nature of the amplitudes then leads to the well-known size-consistency or size-extensivity problem [7]. Another less serious drawback to the CI parametrisation is the lack of manifest normalisation built into it. Thus, $\left\langle\Psi^{1} \mid \Psi\right\rangle \neq 1$ necessarily, al though Eq. (8) does guarantee that $(d / d t)\left\langle\psi^{\prime}(t) \mid \psi(t)\right\rangle=0$ 。 Despite these disadvantages, the CI method has a wide history of successful applications to few-body systems, particularly in the area of quantum chemistry.

\subsection{The NCCM Representation}

The CCM has its origins in the work of Hubbard [24], who realised that the problems associated with the disconnected nature of the CI operator F in Eq. (9), could be overcome by writing $F$ in exponential form, $F=\exp (S)$. He showed that, by contrast to the disconnected nature of $F$, the operator $S$ contains only connected or linked terms. The linked cluster theorem of Goldstone [25] for the ground-state energy also follows from this parametrisation in the usual well-known fashion. 
Often, we are interested only in the ground-state energy eigenvalue $E_{0}$, in which case the static ground-state Schrödinger equation $H|\psi\rangle=E_{0}|\psi\rangle$, may be written in the form [l],

$$
\mathrm{e}^{-\mathrm{S}_{\mathrm{He}} \mathrm{S}}|\Phi\rangle=\mathrm{E}_{0}|\Phi\rangle
$$

The overlap of $E q .(16)$ with the state $<\Phi \mid$ then gives $E_{0}$ itself as a functional of the operator $S$, while the overlaps with the states $\langle\Phi| C_{k}$ give the usual NCCM hierarchy of equations which fully determine the various matrix elements of S. Clearly, in order to calculate the expectation values of operators other than $H$, we need also to parametrise the state $\left\langle\psi^{\prime}\right|$. The NCCM parametrisations of the states $|\Psi\rangle$ and $\left\langle\Psi^{\prime}\right.$ |, which may be compared with their CI counterparts of Eq. (9), are now given as

$$
\begin{aligned}
|\Psi\rangle=e^{S}|\Phi\rangle ; \quad S=\sum_{k}^{1} \sigma_{k}^{2} C_{k}^{\dagger}, \\
\left\langle\Psi^{\prime}\right|=\langle\Phi| \Omega e^{-S} ; \quad \Omega=1+\sum_{k}^{1} \tilde{\sigma}_{k}^{2} C_{k},
\end{aligned}
$$

where the superscript 2 now indicates NCCM quantities. Equation (17) immediately implies the manifest normalisation $\left\langle\Psi^{1} \mid \Psi\right\rangle=1$. The action functional of Eq. (7) now has the NCCH representation,

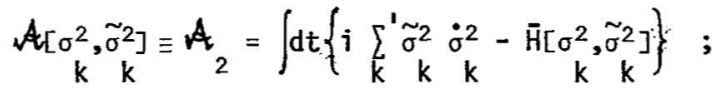

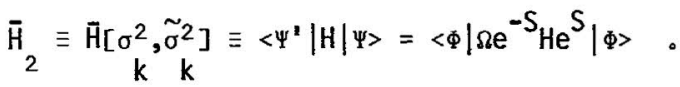

We note that the taking of the derivatives in Eq. (7) is greatly facilated by the fact that the creation and annihilation subalgebras are Abelian.

An average-value functional may now be defined for an arbitrary operator $A$ in the NCCM representation as,

$$
\begin{aligned}
& \left\langle\Psi^{\prime}|A| \Psi\right\rangle \equiv \bar{A}\left[\sigma_{k}^{2}, \tilde{\sigma}_{k}^{2}\right] \equiv \bar{A}_{2}=\langle\Phi| \Omega e^{-S_{A e} S|\Phi\rangle} \\
& \text { N } \\
& =\sum_{n=0}(n !)^{-1}\langle\Phi|\Omega[\cdots[[A, S], S], \cdots S]| \Phi\rangle \text {, }
\end{aligned}
$$

where, in the wel1-known nested commutator expansion employed in the last line of Eq. (20), the operator $S$ appears $n$ times. It is important to realise that if $A$ is a symmetrised sum of k-body operators, the otherwise infinite limit on the sum in this expansion, terminates at the term $N=2 k$ due to the fact that $S$ is composed only of creation operators (defined with respect to the model state $|\Phi\rangle$ ). We may write the formal expansion,

$$
\bar{A}\left[\underset{k}{\left.\sigma^{2}, \tilde{\sigma}^{2}\right]}=\sum_{m=0}^{1} \sum_{n=0}^{N}(m ! n !)^{-1} \sum_{k} \sum_{l}^{1} \cdots \sum_{l_{n}}^{1}<k|A| \ell_{1} \cdots \ell_{n}>\times \underset{k}{\sigma_{2}^{2} \sigma_{l}^{2}} \cdots \sigma_{l_{n}}^{2},\right.
$$


where, by comparison with Eq. (20), the matrix elenents may be written schematically as

$$
\left\langle k|A| \ell_{1} \cdots \ell_{n}>\equiv\left\langle\Phi\left|C_{k} A C_{\ell}^{\dagger} \cdots C_{\ell_{n}}^{\dagger}\right| \Phi\right\rangle_{L},\right.
$$

and where the suffix $\mathcal{L}$ on these matrix elements indicates the linked structure implied by the nested commutator expansion of Eq. (20).

It is clear from Eq. (18) that the stationarity of $A_{2}$ with respect to small variations in the amplitudes $\left\{\sigma^{2}, \widetilde{\sigma}^{2}\right\}$ now gives the NCCM equations of motion of precisely the same form as in the ${ }^{k}{ }_{C I}$ equations (12), but with the CI superscripts $I$ replaced by their NCCM counterparts 2. At the stationary equilibrium, the ground state is again similarly given as in Eq. (13). The NCCM counterparts of these equations have the more explicit form,

$$
\begin{aligned}
& \langle\Phi| C_{k} e^{-S_{H e} S_{\mid \Phi>}=0,}, k \neq 0, \\
& <\Phi\left|\Omega\left[e^{-S_{H e} S}, C_{k}^{\dagger}\right]\right| \Phi>=0 ; k \neq 0,
\end{aligned}
$$

which may be regarded as a set of coupled equations to determine the static amplitudes $\left\{\sigma^{2}, \tilde{\sigma}^{2}\right\}$. It is also not difficult to show that the Dyson equations for these amplitudes $^{k}\left\{\sigma^{2}, \tilde{\sigma}^{2}\right\}$ may again be put in a form precisely analogous to Eq. (15). However, a very imfortant modification arises from the replacement $\bar{v}_{1}+\bar{V}_{2}$ in passing from the CI to the NCCM representation. Thus in the NCCM case the resulting Goldstone energy diagrams are fully connected, as indeed are the diagrams for the set of amplitudes $\left\{\sigma_{\mathrm{k}}^{2}\right\}$ which characterise the operator $\mathrm{S}$. This is clearly to be expected from our earlier discussion on the Goldstone linked-cluster theorem. By contrast, the amplitudes $\left\{\tilde{\sigma}_{\mathrm{k}}^{2}\right\}$ still contain disconnected pieces, and remain problematic. If the only quantity of interest is the ground-state energy $E_{0}$, then these quantities $\left\{\tilde{\sigma}_{k}^{2}\right\}$ are not needed, as may be seen from taking the overlap of Eq.(16) with $\langle\Phi|$, as discussed already above. However, the problem of disconnectedness sti1l remains for the calculation of other quantities.

Very related to the preceeding discussion of the linked-cluster nature of the "bottom" amplitudes $\left\{\tilde{\sigma}_{\mathrm{k}}^{2}\right\}$ is the concept of generalised time ordering (g.t.o.). This has traditionally been a rather useful formal device for classifying and combining certain classes of Goldstone diagrams $[20,26]$. It is based on the factorisation property of Goldstone diagrams containing disjoint sets of legs, which permits a factorisation of the corresponding energy denominators across such sets of legs after all permitted time orderings which preserve these legs are taken together. It turns out that the linked nature of the bottom amplitudes $\left\{\sigma_{\mathrm{k}}^{2}\right\}$ implies that the NCCM generates automatically diagrams for the energy with such a g.t.o. property in the "downward" direction (i.e., backwards in time) only. Conversely, the fact that the "top" amp1itudes $\left\{\tilde{\sigma}_{k}^{2}\right\}$ are still disconnected, implies the absence of a g.t.o. property in the "upward" direction. 
The upshot is that the energy diagrams (i.e., for the expectation value of the Hamiltonian at the stationary equilibrium') which are themselves now all connected, can thereby be represented by what are termed normal g.t.o. trees or NCCM trees. Roughly speaking these are diagrans which "branch out" in the downward direction only (-- and hence represent the root system of a real tree rather than $i$ ts visible branch structure). It has been rather fully explained elsewhere [20] how each link or branch (-- or root) of such a diagram corresponds to a specific set of particle (or particle-hole) lines associated with the configuration-space index $k$ of the corresponding state $C_{k}^{\dagger} \mid \Phi>$. If such a (downward) closed NCCM tree diagram for the energy (-- the totality of which correspond precisely to the usual NCClA expression calculated from the overlap of $\mathrm{Eq}$. (16) with the state $\langle\Phi|$ ) is divided into two open portions by cutting a single link or branch, the bottom part constitutes a diagram associated with some specific amplitude $\sigma_{k}^{2}$ and will always itself be linked; whereas the top part constitutes a corresponding diagram for the amplitude $\tilde{\sigma}_{k}^{2}$, and may be unlinked. The interested reader is referred to the extensive NCCM 1iterature [4-9] for further details.

\subsection{The ECCM Representation}

The major aim of the ECCM is now to cure the remaining disconnectedness in the top amplitudes $\left\{\tilde{\sigma}_{k}^{2}\right\}$, so that the complete formalism may be based on a parametrisation in which all of the amplitudes that completely characterise the description of the system obey the cluster property. The ECCM parametrisation of the states | $\Psi>$ and $\left\langle\psi^{\prime} j\right.$ is now,

$$
\begin{aligned}
|\Psi\rangle & =\mathrm{e}^{S}|\Phi\rangle ; S=\sum_{k}^{1} S_{k} C_{k}^{\dagger}, \\
\left\langle\Psi^{\prime}\right| & =\langle\Phi| \mathrm{e}^{\tilde{\Sigma}} \mathrm{e}^{-S} ; \tilde{\Sigma}=\sum_{k}^{1} \tilde{\sigma}_{k}^{3} C_{k},
\end{aligned}
$$

which may be compared with its CI and NCCM counterparts in Eqs. (9) and (17) respectively. Whereas in the NCCM representation, the coefficients $\tilde{\sigma}_{K}^{2}$ are just the (unlinked) average values of the corresponding configuration-space creation operators $C_{k}^{\dagger}$ (i.e., $\left.\tilde{\sigma}_{k}^{2}=\left\langle\psi^{\prime}\left|C_{k}^{\dagger}\right| \psi\right\rangle\right)$, it can now be shown that in the ECCM representation the corresponding amplitudes $\tilde{\sigma}_{\mathrm{k}}^{3}$ are precisely the linked pieces of these averages which remain after the disconnected terms have been discarded. The ground states maintain their manifest normalisation within the ECCM, $\left\langle\Psi^{1} \mid \psi\right\rangle=1$.

An average-value functional of an arbitrary operator $A$ now takes the form,

$$
\bar{A} \equiv\left\langle\Psi^{1}|A| \Psi\right\rangle=\langle\Phi|\hat{A}| \Phi\rangle,
$$

in terms of the doubly similarity-transformed operator $\hat{A}$,

$$
\hat{A} \equiv e^{\widetilde{\Sigma}} e^{-S} A e^{S} e^{-\tilde{\Sigma}}
$$


It turns out also to be particularly convenient to transform from the set of (linkedcluster) amplitudes $\left\{s_{k}, \tilde{\sigma}_{k}^{3}\right\}$ belonging to the operators $(S, \tilde{\Sigma})$ of Eq. (24) to a new, more symmetric, linked-cluster set $\left\{\sigma_{k}^{3}, \tilde{\sigma}_{k}^{3}\right\}$ belonging to the operators $(\Sigma, \tilde{\Sigma})$ defined as,

$$
\Sigma|\Phi\rangle=(1-|\Phi\rangle\langle\Phi|) \mathrm{e}^{\tilde{\Sigma}} \mathrm{S}|\Phi\rangle ; \Sigma=\sum_{\mathbf{k}}^{\int_{k} \sigma^{3} \mathrm{C}^{\dagger}},
$$

and which hence have the inverse transformations,

$$
\sigma_{k}^{3}=\left\langle\Phi\left|C_{k} e^{\tilde{\Sigma}} S\right| \Phi\right\rangle \quad ; \quad s_{k}=\left\langle\Phi\left|C_{k} e^{-\tilde{\Sigma}} \Sigma\right| \Phi\right\rangle
$$

It is not difficult to show that the action functional of Eq.(7) now has the ECCM representation,

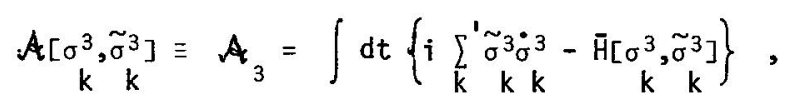

which may be compared with with the CI and NCCM counterparts of Eqs.(10) and (18) respectively. The corresponding expression for $\bar{H}_{3} \equiv \bar{H}\left[\sigma_{k}^{3}, \tilde{\sigma}_{k}^{3}\right]$ is now more complicated than the NCCM expression of Eqs.(19)-(22) due to the presence of the double similarity transformation. However, it can be shown that an average-value functional $\bar{A}$ of an arbitrary operator A now has the ECCM form [20],

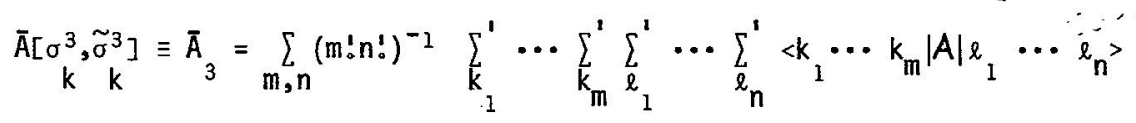

$$
\begin{aligned}
& \times \begin{array}{cccccc}
\tilde{\sigma}^{3} & \cdots & \tilde{\sigma}^{3} & \sigma^{3} & \cdots & \sigma^{3} \\
k_{1} & & k_{m} & l_{1} & & \ell_{n}
\end{array} .
\end{aligned}
$$

The matrix elements in Eq.(30) may be evaluated directly from the definitions of Eqs. $(25)-(28)$. They may be written schematically, by comparison with the NCCM expression of Eq. (22) as,

$$
\left\langle k_{1} \cdots k_{m}|A| \ell_{1} \cdots \ell_{n}\right\rangle \equiv\left\langle\Phi\left|C_{k_{1}} \cdots C_{k_{m}}^{A C} C_{l}^{\dagger} \cdots C_{\ell_{n}}^{\dagger}\right| \Phi\right\rangle \mathcal{L}^{\prime},
$$

in which the suffix $D \mathcal{L}$ indicates the definite or double linking structure implied by their definition. It has been shown elsewhere in detail [20] that the D\&-linking is characterised by the following two constraints: (i) Erom each group of particles characterised by each of the configuration-space indices $\ell$, the amplitude $\sigma_{\ell}^{3}$ associated with the creation operator $C_{l}^{\dagger}$, must have at least one particle line connected to the operator $A$ (as in the $\mathcal{L}-$ linking of the NCCM); and further (ii) For each group of particles characterised by each of the configuration space indices $k$, the amplitude $\tilde{\sigma}^{3}$ associated with the destruction operator $C_{k}$, must either have at least one particle line connected to the operator $A$, or there must be connections, 
by at least one particle line in each case, to at least two separate amplitudes $\sigma_{\ell}^{3}$ associated with two different creation operators $C_{\ell}^{\dagger}$ in the matrix element of Eqs. (30) - (31).

It is also clear from Eq. (29) that the stationarity of $\mathcal{A}_{3}$ with respect to small variations in the independent amplitudes $\left\{\sigma_{k}^{3}, \tilde{\sigma}_{k}^{3}\right\}$ now again gives the ECCN equations of motion of precisely the same form as their CI counterparts of Eq. (12) but with the CI superscripts 1 replaced by the ECCH counterparts 3 . Once again, the energy functional $\tilde{H}\left[\sigma_{k}^{3}, \tilde{\sigma}_{k}^{3}\right] \equiv \bar{H}$ plays the role of determing the dynamics of the basic ECCM amplitudes ${ }_{\left\{\sigma_{k}^{3}, \tilde{\sigma}^{3}\right\}}{ }^{3}$ and their stationary equilibrium values. After a little algebra it can be shown that the kinetic energy operator $T$ has an ECCli average value functional which can be expressed in a very similar form to its CI counterpart of Eq. (14),

$$
\begin{aligned}
\underset{k}{\overline{\mathrm{T}}}\left[\sigma_{k}^{3}, \tilde{\sigma}^{3}\right] \equiv \overline{\mathrm{T}}_{3} & =\langle\Phi|\tilde{\Sigma} T \Sigma| \Phi\rangle \\
& =\sum_{k}^{1} \mathrm{e}_{k} \tilde{\sigma}_{k}^{3} \sigma^{3}+\text { const. }
\end{aligned}
$$

The Dyson equations for the ECCM amplitudes $\left\{\sigma_{k}^{3}, \tilde{\sigma}_{k}^{3}\right\}$ may therefore once again be put into a form precisely analogous to Eq. (15). Once again, these may be iterated to show that the equilibrium values of the ECCM amplitudes $\left\{\sigma^{3}, \tilde{\sigma}^{3}\right\}$ may be classified in terms of definite classes of Goldstone diagrams, which are now denoted as the extended g.t.o. trees or ECCM trees [20]. The detailed rules for their construction have been given elsewhere [20].

The main difference from the downward-branching NCCM tree diagrams, is that the ECCM tree diagrams for the energy now branch upwards as well as downwards from any vertex $V$. Furthermore, the average value functional $\bar{A}\left[\sigma^{3}, \tilde{\sigma}^{3}\right]$ of an arbitrary operator in $H$ may be uniquely expressed in terms of such $\mathrm{K}_{E C C} \mathrm{k}$ tree structures. A particular consequence of the g.t.o. structure in both time directions for the ECCM trees, is that if any such (closed) ECCM tree diagram for the energy is divided into two by cutting any single link labelled by some configuration-space index $k$, then both the top part (which is a diagram that contributes to $\tilde{\sigma}_{k}^{3}$ ) and the bottom part (which is a contributing diagram to $\sigma^{3}$ ) remain individually connected. This is a reflection of the fact that the ECCM has succeeded in finding a complete parametrisation of the many-body system, in which alz of the basic amplitudes $\left\{\sigma^{3}, \tilde{\sigma}^{3}\right\}$ that in their totality exactly describe the system, are quasi-local in the sense of obeying the cluster property. This feature is expected to be of considerable importance for descriptions of global properties of the many-body system, for the incorporation of such effects as topological excitations or deformations, or for a proper description of a phase or shape transition in the system.

Having now completed our comparison of the ECCM with the better known CI method and the NCCM, we now deal henceforth only with the ECCM parametrisation. We therefore now drop the suffices and superscripts 3 , and rename the basic amplitudes $\left\{\sigma_{k}^{3}, \tilde{\sigma}_{k}^{3}\right\}$ 
$\sim\left\{\sigma_{k}, \tilde{\sigma}_{k}\right\}$, for example.

\section{ECCM AVERAGE VALUES AND RELATED MATRIX ELEMENTS}

We have seen explicitly that neither the NCCM nor the ECCM parametrisations of the many-body problem manifestly preserve the hermiticity of physical operators. In the ECCN this ultimately derives from the lack of unitarity of the double similarity transformations which are generated by the bra and ket state parametrisations, as in Eqs. (25) and (26). The basic formalism is thus a biorthogonal formulation of the many-body problem rather than an orthogonal one, and the average-value functional $\bar{A}$ of an arbitrary operator A given by Eqs. (25) and (26) represents the real expectation value in the exact ground state in view of the manifest normalisation, $\left\langle\Psi^{2} \mid \psi\right\rangle=$ 1.

Further progress is most readily made by developing the ECCM to express the various matrix elements that arise in practice which involve the doubly similaritytransformed arbitrary operator $\hat{A}$ of Eq. (26) in terms of the basic ECCM amplitudes $\left\{\sigma_{k}, \tilde{\sigma}_{k}\right\}$. This is most compactly done in terms of functional derivatives of the average-value functional $\bar{A}=\bar{A}\left[\sigma_{k}, \tilde{\sigma}_{k}\right]$ itself. We first note that these functional derivatives, and hence also the matrix elements of Eq. (31), are not all independent. For example, it is trivial to see that, when expressed in terms of the original operators $(S, \widetilde{\Sigma})$ or their configuration-space amplitudes $\left\{s_{k}, \tilde{\sigma}_{k}\right\}$ of $E q .(24)$, the average value functional

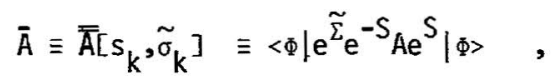

satisfies the relation,

$$
\frac{\delta^{2} \overline{\bar{A}}}{\delta \tilde{\sigma}_{k} \delta \tilde{\sigma}_{\ell}}=\left\langle\Phi\left|C_{k} C_{\ell} \hat{A}\right| \Phi\right\rangle=\frac{\delta \overline{\bar{A}}}{\delta \tilde{\sigma}_{k+\ell}} \text {, }
$$

where we have used the compact notation for the compounding of configuration-space indices introduced in Sec. 2. By the usual rules of partial differentiation involved in changing variables to the new linked-cluster amplitudes $\left\{\sigma_{k}, \tilde{\sigma}_{k}\right\}$, with $\bar{A}\left[s_{k}, \tilde{\sigma}_{k}\right] \equiv$ $\bar{A}\left[\sigma_{k}, \tilde{\sigma}_{k}\right]$, it is not difficult to show that the trivial identity of Eq. (34) becomes the relation,

$$
\begin{aligned}
\frac{\delta \bar{A}}{\delta \tilde{\sigma}_{k+\ell}}=\frac{\delta^{2} \bar{A}}{\delta \tilde{\sigma}_{k} \delta \tilde{\sigma}_{\ell}} & +\sum_{m}^{\prime}\left(\sigma_{k+m} \frac{\delta^{2} \bar{A}}{\delta \sigma_{m} \delta \tilde{\sigma}_{\ell}}+\frac{\delta^{2} \bar{A}}{\delta \tilde{\sigma}_{k} \delta \sigma_{m}} \sigma_{m+\ell}\right) \\
& +\sum_{m}^{i} \sum_{n}^{\prime} \sigma_{k+m} \frac{\delta^{2} \bar{A}}{\delta \sigma_{m} \delta \sigma_{n}} \sigma_{n+\ell}
\end{aligned}
$$

Now, we shall also be interested in the average values of products of operators

$$
\left\langle\Psi^{1}\left|A_{1} A_{2} \cdots A_{n}\right| \Psi\right\rangle=\left\langle\Phi\left|\hat{A}_{1} \hat{A}_{2} \cdots \hat{A}_{n}\right| \Phi\right\rangle \quad,
$$


and the easiest way to calculate such products is by the insertion of a resolution of the identity operator of the form of Eq. (2) between each pair of double similaritytransformed operators in the matrix element on the right-hand side of Eq. (36). The remaining matrix elements may then be related to the average-value functionals $\vec{A}_{j}$ of the individual operators in the product by taking similar suitable functional derivatives. As above, it is easy to show that in terms of the original ECCM amplitudes $\left\{s_{k}, \tilde{\sigma}_{k}\right\}$ we have the first-order functional derivatives,

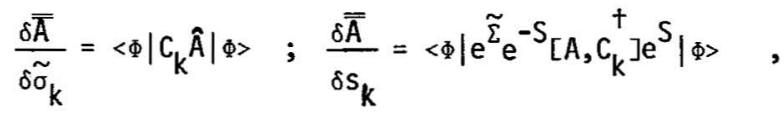

which may then be re-expressed in terms of the final ECCM amplitudes $\left\{\sigma_{k}, \tilde{\sigma}_{k}\right\}$ by similar use of the chain rule of partial differentiation. In this manner it is not difficult to prove the relations,

$$
\left\langle\Phi\left|C_{k} \hat{A}\right| \Phi\right\rangle=\frac{\delta \bar{A}}{\delta \tilde{\sigma}_{k}}+\sum_{\ell}^{\prime} \sigma_{k+\ell} \frac{\delta \bar{A}}{\delta \sigma_{\ell}} ; k \neq 0,
$$

and

$$
\left\langle\Phi\left|\hat{A} C_{k}^{\dagger}\right| \Phi\right\rangle=\frac{\delta \bar{A}}{\delta \sigma_{k}}+\sum_{l}^{\prime} \frac{\delta \bar{A}}{\delta \tilde{\sigma}_{\ell}} L_{j k}+\sum_{m}^{\prime} \sum_{n}^{\prime} \frac{\delta \bar{A}}{\delta \sigma_{m}} \sigma_{m+n} L_{n k} ; k \neq 0,
$$

where the amplitude $L_{k \ell}=L_{\ell k}$ is defined (for $k \neq 0 \neq \ell$ ) as

$$
L_{k \ell} \equiv \sum_{m} \sum_{n} \omega_{m+n} \bar{\omega}_{k-m} \bar{\omega}_{\ell-n}=\langle\Phi| e_{k \ell}^{\tilde{\Sigma}_{c} c^{\dagger} c^{\dagger}|\Phi\rangle}{ }_{D \ell}
$$

in terms of the more basic amplitudes $\omega_{k}$ and $\bar{w}_{k}$ defined themselves as,

$$
\omega_{k} \equiv\left\langle\Phi\left|e^{\tilde{\Sigma}} C_{k}^{\dagger}\right| \Phi\right\rangle \quad ; \quad \bar{\omega}_{k} \equiv\left\langle\Phi\left|e^{-\tilde{\Sigma}_{c}^{\dagger}} C_{k}^{\dagger}\right| \Phi\right\rangle \quad
$$

Further details of the derivations of Eqs. (38) and (39) are given elsewhere [21], together with similar expressions for such higher matrix elements as $\left\langle\Phi\left|C_{k} \hat{A C}_{\ell}^{\dagger}\right| \Phi\right\rangle$ which will correspondingly involve second-order functional derivatives. We note here only that the derivation of such expressions is made simpler by suitable use of the Wick theorem in the form of Eq. (6), and by judicious insertions of the identity operator in the form of Eq. (2).

As an example of the use of Eqs. (38) and (39), we compute an expression for the particularly important example of the average value $\overline{A B} \equiv\left\langle\Psi^{\prime}|A B| \psi\right\rangle$ of the product of two operators $A$ and $B$. By an insertion of the identity operator as discussed immediately below Eq.(36), imnediate use of Eqs. (38) and (39) leads to the result

$$
\overline{\mathrm{AB}}=\overline{\mathrm{A}} \cdot \overline{\mathrm{B}}+\sum_{\mathrm{k}}^{\prime} \sum_{\ell}^{\prime}\left(\frac{\delta \mathrm{A}}{\delta \sigma_{\mathrm{k}}} \frac{\delta \overline{\mathrm{B}}}{\delta \sigma_{\ell}} \mathrm{X}_{\mathrm{k} \ell}^{11}+\frac{\delta \overline{\mathrm{A}}}{\delta \sigma_{\mathrm{k}}} \frac{\delta \overline{\mathrm{B}}}{\delta \tilde{\sigma}_{\ell}} \mathrm{X}_{\mathrm{k} \ell}^{12}\right.
$$




$$
\left.+\frac{\delta \bar{A}}{\delta \tilde{\sigma}_{k}} \frac{\delta \bar{B}}{\delta \sigma_{\ell}} X_{k \ell}^{21}+\frac{\delta \bar{A}}{\delta \tilde{\sigma}} \frac{\delta \bar{B}}{\delta \tilde{\sigma}} X_{k}^{22} k_{\ell}\right),
$$

where the various $X$-coefficients are defined as,

$$
\begin{aligned}
& \underset{k \ell}{x^{11}}=x_{\ell k}^{11} \equiv \sigma_{k+\ell}+\sum_{m}^{\prime} \sum_{n}^{\prime} \sigma_{k+m} L_{m n} \sigma_{n+l}, \\
& \underset{k \ell}{X^{12}} \equiv \delta(k, \ell)+\sum_{m}^{1} \sigma_{k+m} L_{m \ell}, \quad \frac{x^{21}}{k \ell} \equiv \sum_{m}^{\prime} L_{k m}{ }^{\sigma} m+l, \\
& \mathrm{x}_{\mathrm{k} \ell}^{22}=\underset{\ell \mathrm{k}}{\mathrm{x}^{22}} \equiv \mathrm{L}_{\mathrm{k} \ell}
\end{aligned}
$$

Similar expressions may also be derived for the average values of the products of more than two operators.

\section{GENERALISED POISS ON BRACKETS AND AN EXACT CLASSICAL MAPPING}

It is of particular interest to apply the results of Eqs. (42) and (43) above to the average value of the commutator of the two arbitrary operators $A$ and $B$. In view of the very high degree of symmetry exhibited by the X-coefficients of $E q .(43)$, the result simplifies greatly in comparison to the results for either of the individual products $\overline{A B}$ or $\overline{B A}$. We find,

$$
\left\langle\underline{\Psi}^{\prime}|A B-B A| \Psi\right\rangle \equiv\langle[A, B]\rangle=i\{\bar{A}, \bar{B}\} \quad,
$$

where the quantity $\{\bar{A}, \bar{B}\}$ has the form,

$$
i\{\bar{A}, \bar{B}\} \equiv \sum_{k}^{\prime}\left(\frac{\delta \bar{A}}{\delta \sigma_{k}} \frac{\delta \bar{B}}{\delta \tilde{\sigma}_{k}}-\frac{\delta \bar{B}}{\delta \sigma_{k}} \frac{\delta \bar{A}}{\delta \tilde{\sigma}_{k}}\right),
$$

of a generalised classical Poisson bracket, and where the imaginary factor $i$ has been inserted for convenience of comparison.

These results may be made even more suggestive of a generalised classical Hamiltonian mechanics by defining as new quasi-local variables the generalised configuration-space fields $\phi_{k}$ and their canonically conjugate generalised momentum densities $\pi_{k}$, defined as the linear combinations,

$$
\phi_{k} \equiv 2^{-\frac{1}{2}}\left(\sigma_{k}+\tilde{\sigma}_{k}\right) ; \pi_{k} \equiv 2^{-\frac{1}{2}} i\left(\tilde{\sigma}_{k}-\sigma_{k}\right)
$$

An arbitrary average value functional may now be re-expressed in terms of these new amplitudes, $\angle A>\equiv \bar{A} \rightarrow \bar{A}\left[\phi_{k}, \pi_{k}\right]$, and Eq. (45) may then be rewritten as,

$$
\{\bar{A}, \bar{B}\}=\sum_{k}^{\prime}\left(\frac{\delta \bar{A}}{\delta \phi_{k}} \frac{\delta \bar{B}}{\delta \pi_{k}}-\frac{\delta \bar{B}}{\delta \phi_{k}} \frac{\delta \bar{A}}{\delta \pi_{k}}\right) \text {. }
$$


Furthermore, the ECCM equations of motion which follow from the stationarity of the action functional of Eq. (29) with respect to small variations in the independent amplitudes $\left\{\sigma_{k}, \tilde{\sigma}_{k}\right\}$, may also be re-expressed in terms of the new quantities as,

$$
\frac{d \phi_{k}}{d t}=\left\{\phi_{k}, \bar{H}\right\}=\frac{\delta \bar{H}}{\delta \pi_{k}} ; \frac{d \pi_{k}}{d t}=\left\{\pi_{k}, \bar{H}\right\}=-\frac{\delta \hat{H}}{\delta \phi_{k}} .
$$

Finally, it is easy to show using Eqs. (48), that an arbitrary, intrinsically timedependent operator $A=A(t)$, has an average-value functional $\bar{A}=\bar{A}\left[\phi_{k}, \pi_{k} ; t\right]$ with an equation of motion given by,

$$
\frac{d \bar{A}}{d t}=\frac{\overline{\partial A}}{\partial t}+\{\bar{A}, \bar{H}\}=\langle\partial A / \partial t\rangle+\frac{1}{i}\langle[A, H]\rangle,
$$

where the second equality of Eq. (49) is just the expectation value of the usual quantum-mechanical Heisenberg equation of motion. Equation (49) may be understood as an exact and well-defined generalisation of the correspondence principle or Ehrenfest theorem.

In this way we are led to the very important result that the ECCM parametrisation has formally exactly mapped the original quantur many-body problem onto the (generalised) nonlocal classical Hamiltonian mechanics of the c-number, quasi-local, linkedcluster fields $\left\{\phi_{k}, \pi_{k}\right\}$ or $\left\{\sigma_{k}, \tilde{\sigma}_{k}\right\}$. These fields are themselves defined as manybody amplitudes in the configuration space labelled by the indices $k$ which describe the particular subsets of particles under consideration. This exact mapping opens up the possibility of being able to exploit or to extend all of the techniques developed in classical mechanics for use in the quantum many-body problem. For example, one can make easy contact with conservation lows and the associated sum miles by using the Noether currents. It is intuitively apparent from the existence of this mapping onto classical mechanics that the c-number ECCM amplitudes $\left\{\phi_{k}, \pi_{k}\right\}$ or $\left\{\sigma_{k}, \tilde{\sigma}_{k}\right\}$ which completely characterise and decompose our many-body problem, may be viewed as a set of generalised mean fields which describe each subsystem of particles in the interacting many-body system as labelled by the configuration-space indices $\{k\}$. Indeed, this interpretation has been formally extended by us [21] to show how the ECCM may very profitably also be viewed as an exact bosonisation procedure in which the ECCM states may be exactly associated in a one-to-one fashion with a set of generalised coherent states in some suitably defined boson space. The usual Glauber coherent states are seen to be just the associates of the lowest-order one-body mean fields $\sigma_{1}(\vec{x})$ and $\tilde{\sigma}_{1}(\vec{x})$. This ECCM bosonisation procedure differs from other more wellknown such procedures by taking the usual motivation for any bosonisation scheme to its ultimate conclusion -- namely, that the resultant generalised coherent boson fields are simply classical c-number fields with only classical (nonlinear) interactions between them. He believe that this further re-interpretation of the ECCM as a generalised bosonisation procedure in which generalised coherent states may be 
defined, may also be profitably exploited to develop new approximation schemes for handling quantum many-body systems. Rather than pursuing this line of approach further here, we refer the interested reader to Ref. [21] for further details, and turn our attention instead to a description of excited states within the ECCM.

\section{AN ECCM DESCRIPTION OF EXCITED STATES}

So far we have focussed mainly on the ground state of the quantal system under discussion, and its dynamic evolution. It is quite possible to extend the groundstate ECCM discussed above to include excited states [22], very much in analogy to the way Emrich [27] has developed the NCCM formalism. We sketch this extension below, and point out particulary the intimate connection of the excited states with small oscillations around the stationary ground-state configurations obtained by the formalism presented previously.

\subsection{The Direct Approach: The Excited State Description}

We now consider the time-independent Schrödinger equation for both the ground and excited states respectively,

$$
\begin{aligned}
& H|\Psi\rangle=E_{0}|\Psi\rangle ;\left\langle\Psi^{\prime}\right| H=E_{0}\left\langle\Psi^{\prime}\right|, \\
& H\left|\Psi_{\lambda}\right\rangle=\left(E_{0}+\varepsilon_{\lambda}\right)\left|\Psi_{\lambda}\right\rangle ; \quad\left\langle\Psi_{\lambda}^{\prime}\right| H=\left(E_{0}+\varepsilon_{\lambda}\right)\left\langle\Psi_{\lambda}^{\prime}\right|,
\end{aligned}
$$

where $\varepsilon_{\lambda}$ is the (positive) excitation energy of the particular excited state denoted by the label $\lambda$. Our ECCM parametrisations for the excited bra and ket eigenstates are now given as,

$$
\begin{aligned}
& \left|\Psi_{\lambda}\right\rangle=e^{S} e^{-\widetilde{\Sigma}_{x} x^{\lambda}}|\Phi\rangle ; x^{\lambda}=\sum_{k}^{1} x_{k}^{\lambda} C_{k}^{\dagger}, \\
& \left\langle\Psi_{\lambda}^{\prime}\right|=\langle\Phi| Y^{\lambda} \cdot e^{\widetilde{\Sigma}} e^{-S} ; Y^{\lambda}=\sum_{k}^{1} y_{k}^{\lambda} C_{k},
\end{aligned}
$$

which may be compared with the ground-state counterparts of Eq.(24). The new excitation and de-excitation operators $x^{\lambda}$ and $y^{\lambda}$ respectively are again composed only of creation and destruction operators respectively with respect to the reference state $\mid \Phi>$. In terms of the parametrisations of Eq. (51), the exicted-state Schrödinger equations of Eq.(50) may equivalently be written in terms of the doubly similaritytransformed Hamiltonian $\hat{\mathrm{H}}$, defined by Eq.(26), as

$$
\hat{H} X^{\lambda}|\Phi\rangle=\left(E_{0}+\varepsilon_{\lambda}\right) X^{\lambda}|\Phi\rangle \quad ; \quad\langle\Phi| Y^{\lambda} \hat{H}=\left(E_{0}+\varepsilon_{\lambda}\right)\langle\Phi| Y^{\lambda},
$$

or, by combining with their ground-state counterparts, as

$$
\left[\hat{H}, X^{\lambda}\right]|\Phi\rangle=\varepsilon_{\lambda} X^{\lambda}|\Phi\rangle \quad ; \quad\langle\Phi|\left[Y^{\lambda}, \hat{H}\right]=\varepsilon_{\lambda}\langle\Phi| Y^{\lambda} .
$$

Equations (52) may be written directly as (scalar) linear eigenvalue equations by 
projecting respectively with the states $\langle\Phi| C_{k}$ and $C_{k}^{\dagger}|\Phi\rangle$. In this way, we obtain the eigenvalue equations,

$$
\begin{aligned}
& \sum_{\ell}^{\prime}\left\langle\Phi\left|C_{k} \hat{H C}{ }_{\ell}^{\dagger}\right| \Phi>x_{\ell}^{\lambda}=\left(E_{0}+\varepsilon_{\lambda}\right) x_{k}^{\lambda},\right. \\
& \sum_{\ell}^{\prime} y_{\ell}^{\lambda}<\Phi\left|C_{\ell} \hat{H C}{ }_{k}^{\dagger}\right| \Phi>=\left(E_{0}+\varepsilon_{\lambda}^{\prime}\right) y_{k}^{\lambda} .
\end{aligned}
$$

The matrix elements in Eq. (54) may now be evaluated by similar techniques as described in Sec. 4 which led to the comparable Eqs. (38) and (39). The matrix elements in Eq. (54) wi1l involve both first- and second-order functional derivatives of the average value functional $\bar{H}$. However we may now use the fact that at the stationary ground-state equilibrium, the first-order functional derivatives vanish, $\delta \bar{H} / \delta \sigma_{k}=0=\delta \bar{H} / \delta \tilde{\sigma}_{k}$. In this way only the second-order functional derivatives survive. A certain amount of algebra [21] shows that at the stationary ground-state equilibrium, we have (for $i \neq 0 \neq j$ ),

$$
\left\langle\Phi\left|C_{i} \hat{H} C_{j}^{\dagger}\right| \Phi\right\rangle=E_{o} \delta(i, j)+E_{i j}+\sum_{m}^{\prime} \sigma_{i+m} F_{m j},
$$

where we have introduced the various second-order functional derivatives of $\vec{H}=$ $\bar{H}\left[\sigma_{k}, \tilde{\sigma}_{k}\right]$ as,

$$
E_{i j} \equiv \frac{\delta^{2} \bar{H}}{\delta \tilde{\sigma}_{i} \delta \sigma_{j}} ; F_{i j} \equiv \frac{\delta^{2} \bar{H}}{\delta \sigma_{i} \delta \sigma_{j}} ; \quad F_{i j} \equiv \frac{\delta^{2} \bar{H}}{\delta \tilde{\sigma}_{i} \delta \tilde{\sigma}_{j}} \text {. }
$$

In this way we find that Eqs. (54) may be written as,

$$
\begin{aligned}
& \sum_{\ell}^{1}\left[E_{k \ell}+\sum_{m}^{\prime} \sigma_{k+m} F_{m \ell}-\varepsilon_{\lambda} \delta(k, \ell)\right] x_{\ell}^{\lambda}=0 ; k \neq 0, \\
& \sum_{\ell}^{\prime} y_{\ell}^{\lambda}\left[E_{\ell k}+\sum_{m}^{\prime} \sigma_{\ell+m} F_{m k}-\varepsilon_{\lambda} \delta(k, \ell)\right]=0 ; k \neq 0 .
\end{aligned}
$$

Equations (57) are just a set of linear eigenvalue equations, in which the excitation energies appear directly as eigenvalues. We note also that our bra and ket excited eigenstates are orthogonal since

$$
\begin{aligned}
\left\langle\Phi\left|Y^{\lambda} \hat{H} X^{\lambda^{\prime}}\right| \Phi\right\rangle & =\left(E_{0}+\varepsilon_{\lambda}\right)\left\langle\Phi\left|Y^{\lambda} X^{\lambda^{\prime}}\right| \Phi\right\rangle \\
& =\left(E_{0}+\varepsilon_{\lambda^{\prime}}\right)\left\langle\Phi\left|Y^{\lambda} X^{\lambda^{\prime}}\right| \Phi\right\rangle .
\end{aligned}
$$

Thus, if $\varepsilon_{\lambda} \neq \varepsilon_{\lambda}$ ', the matrix element on the right-hand side of Eq.(58) must be zero. We may therefore choose the normalisation to satisfy

$$
\left\langle\Psi_{\lambda}^{\prime} \mid \Psi_{\lambda^{\prime}}\right\rangle=\left\langle\Phi\left|Y^{\lambda} X^{\lambda}\right| \Phi\right\rangle=\delta_{\lambda \lambda^{\prime}}
$$

In this way we see that the ECCM has achieved a complete biorthonormal descrip- 
tion of the excitation spectrum. The linked cluster structure is again taken into account as fully as possible. The ECCM parameters $\left\{\sigma_{k}, \tilde{\sigma}_{k}\right\}$ have only to be determined once for the ground-state parametrisation, and thereafter are simply input to the excited-state formalism. The remaining problen for the excited states is a simple diagonalisation of a certain effective Hamiltonion matrix, composed of secondorder functional derivatives of the ground-state average value energy functional $\bar{H}$ evaluated at the stationary equilibrium point. This is just a linear homogeneous eigenvalue problem, and is of the same form for the entire excitation spectrum which is yielded in principle at a single pass. The hope is that since the important correlations have already been incorporated via the ground-state exponential correlation operators, the remaining operators $X^{\lambda}$ and $y^{\lambda}$ have the potential of being relatively simple, at least so far as the important low-lying excitations are concerned.

\subsection{An Indirect Approach: Dynamic Linear Response}

We may also consider the dynamics of small oscillations about the stationary equilibrium point. By writing,

$$
\sigma_{k}(t)=\sigma_{k}^{0}+\delta \sigma_{k}(t), \quad \tilde{\sigma}_{k}(t)=\tilde{\sigma}_{k}^{0}+\delta \tilde{\sigma}_{k}(t),
$$

where $\left\{\sigma_{k}^{0}, \sigma_{k}^{0}\right\}$ now represent the equilibrium values of the basic amplitudes which satisfy the stationary equations,

$$
\delta \overline{\mathrm{H}} / \delta \sigma_{\mathrm{k}}=0=\delta \overline{\mathrm{H}} / \delta \tilde{\sigma}_{\mathrm{k}},
$$

we may now linearise the ECCH equations of motion, which are the analogues of the CI equations (12), keeping only first-order terms in the deviations $\left\{\delta \sigma_{k}(t), \delta \tilde{\sigma}_{k}(t)\right\}$. Using Eq.(61) we find that the small oscillations are governed by an effective Hamiltonian which is constructed out of the second-order functional derivatives of Eq.(56). It is trivial to show that these linearised equations of motion are given explicitiy as,

$$
\begin{aligned}
& i \frac{d}{d t}\left(\delta \sigma_{k}\right)=\sum_{\ell}^{1}\left(E_{k \ell} \delta \sigma_{\ell}+F_{k \ell} \delta \tilde{\sigma}_{\ell}\right), \\
& i \frac{d}{d t}\left(\delta \tilde{\sigma}_{k}\right)=\sum_{\ell}^{1}\left(-F_{k \ell} \delta \sigma_{\ell}-E_{\ell k} \delta \tilde{\sigma}_{\ell}\right) .
\end{aligned}
$$

By writing,

$$
\delta \sigma_{k} \equiv \xi_{k} e^{-i \varepsilon t}, \delta \tilde{\sigma}_{k} \equiv n_{k} e^{-i \varepsilon t},
$$

the equations of motion (62) can be written in an obvious matrix notation as,

$$
H_{D}\left(\begin{array}{l}
\xi(\varepsilon) \\
\eta(\varepsilon)
\end{array}\right)=\varepsilon\left(\begin{array}{l}
\xi(\varepsilon) \\
n(\varepsilon)
\end{array}\right) \text {, }
$$

and the eigenfrequencies $\varepsilon$ of the small oscillations are thereby obtained by diagonalising the effective Hamiltomian or dynamic matrix, $\mathrm{H}_{D^{3}}$, which is given in an obvi- 
ous block matrix form as,

$$
H_{D} \equiv\left(\begin{array}{cc}
E & \tilde{F} \\
-F & -E^{\top}
\end{array}\right)=\left(\begin{array}{cc}
0 & I \\
-I & 0
\end{array}\right)\left(\begin{array}{ll}
F & E^{\top} \\
E & \tilde{F}
\end{array}\right),
$$

where $E^{\top}$ indicates the transpose of the matrix $E \equiv\left(E_{i j}\right)$. The dynamic matrix is clearly seen from Eq. (65) to be capable of being written as the product of an antisymmetric matrix with a symmetric matrix. (We note that the symmetry of the last matrix in Eq.(65) follows from the fact that $F_{i j}=F_{j i}$ and $\tilde{F}_{i j}=\tilde{F}_{j i}$ by definition.) This product form further implies [28] that the characteristic polynomial of $H_{D}$ is an even function of $\varepsilon$, and hence that the eigenvalues $\varepsilon$ must appear in pairs $\pm\left|\varepsilon_{n}\right|$.

This whole analysis, and particularly the form of the dynamic matrix in Eq.(65), is strongly reminiscent of the well-known rondom phase approximation (RPA) of Bohm and Pines $[29,30]$. Indeed, what we have achieved here may be regarded as an exact generalised random phase approximation. There are two major differences from ordinary RPA. Firstly, the indices $\{i\}$ are now configuration-space indices which involve arbitrarily many single-particle degrees of freedom, whereas in ordinary RPA the indices are restricted to single particles (or single particle-hole pairs for fermions). The ordinary RPA is thus the linear dynamics of the usual one-body mean field, whereas we have generalised to the small-amplitude dynamics of the complete set of generalised mean fields $\left\{\sigma_{k}, \tilde{\sigma}_{k}\right\}$ which completely and exactly parametrise the quantum many-body problem in the ECCM. Secondly, the present generalised RPA is still exact so long as it is not truncated.

Finally, we note that it is possible to prove that the eigenfrequencies $\left|\varepsilon_{n}\right|$ of the small oscillations found by diagonalising the dynamic matrix $H_{D}$, are identical to the eigenvalues $\varepsilon_{\lambda}$ of the direct approach above to excited states. The proof is not trivial and we do not reproduce it here. It is also clear that one may go beyond the harmonic approximation pertinent to the small oscillations (or linear response) considered here. Thus we may express the complete energy functional $\bar{H}\left[\sigma_{k}, \tilde{\sigma}_{k}\right]$ in terms of the normal modes of the quadratic effective Hamiltonian. The original quantum many-body problem is thereby again mapped into a classical field theory with nonitinear (or anharmonic) couplings of arbitrarily high order between the normal modes in the ECCM configuration space. This procedure has the added advantage of making explicit the hermiticity of the energy functional $\bar{H}$, which up until now has not been manifest when expressed in terms of the original amplitudes $\left\{\sigma_{k}, \tilde{\sigma}_{k}\right\}$, due to the underlying non-unitary nature of the basic ECCM double similarity transformation. The interested reader may find further details of the excited-state ECCM in Ref. [22].

\section{CONCLUDING REMARKS}


We have shown how by applying the ECCM to an arbitrary quantum-mechanical problem with a Schrödinger dynamics, the entire problem may in some definite sense be made classical. In particular, we have mapped the quantal problem into a classical field theory for a set of configuration-space indexed, many-body, quasi-local, classical (i.e., c-number) fields $\left\{\sigma_{k}, \tilde{\sigma}_{k}\right\}$, which interact via nonlocal classical interactions. We have shown how these ECCM amplitudes may further be viewed as a set of generalised mean many-body fields, by considering the dynamics of their small oscillations around the local stationary equilibrium point. We have further seen how this procedure leads to an exact generalisation of RPA, and also how the eigenfrequencies of these normal modes for the generalised mean fields map one-to-one exactly onto the excitation energies of the excited states of the system.

Our ECCM approach may also profitably be compared with the density-functional approach of Hohenberg, Kohn and Sham [31]. Density-functional theory (DFT) attempts to construct the total energy of the system as a functional of a local parameter, namely the particle number density $\rho(\vec{x})$. In its simplest forms, such as the ThomasFermi approximation, DFT gives the total energy as a completely local functional of $\rho(\vec{x})$. Most of the later effort in DFT has gone into attempts to construct suitable nonlocal extensions to these simple local approximations. Within this framework, we may view the ECCM as providing a rather different means for incorporating the nonlocal corrections in a precise and formally exact fashion.

Although the ECCM is in principle exact, in practical applications approximations or truncations will naturally have to be made. The g.t.o. properties that we have discussed in Sec. 3 assume particular significance in this regard. One consequence is that even at a low level of truncation, every low-order Goldstone perturbation theory diagram is always kept together with an infinite set of other diagrams corresponding to terms of arbitrarily high order in perturbation theory. For example, a typical truncation scheme is the so-called SUBn scheme in which the configuration-space indices $\{k\}$ are restricted to involve at most $n$ particles (or particle-hole pairs for fermions). Thus, in the ECCM the SUB1 approximation is just classical mean field theory (i.e., the (Glauber) coherent-state approximation for bosons or Hartree-Fock theory for fermions), whereas the SUB2 approximation for bosons is equivalent to the Gaussian approximation of quantum field theory. Whereas the truncation index $n$ would typically need to be quite large for good accuracy in a CI calculation of a typical non-trivial problem where low-order perturbation theory is insufficient, both the NCCM and ECCM can usually achieve comparable accuracy with much lower values of n. It is this feature which ultimately enables the ECCM to surpass its distant origins in perturbation theory, and which gives it the potential to describe such phenomena as phase or shape transitions and topological excitations, which would be impossible to describe by finite-order perturbation theory.

We believe that the ECCM has so many attractive features as to make it a potentially very powerful addition to the rather limited number of fundamental tools with which we may attack quantum many-body problems. In a companion article in this 
volume we illustrate this potential by applying the ECCM to the problem of quantum fluid dynamics

\section{ACKNOWLEDGEMENT}

One of us (RFB) wishes to acknowlege the support of a research grant from the Science and Engineering Research Council of Great Britain.

\section{REFERENCES}

1 Coester $F$ (1958) Nucl Phys $\underline{7}: 421$

2 Coester F and Kümmel H (1960) Nucl Phys 17: 477

3 Čižek J (1966) J Chem Phys 45:4256; Adv Chem Phys (1969) 14:35

4 Kümmel H, Lührmann K H and Zabolitzky J G (1978) Phys Rep 36C:1

5 Bishop R F and Lührmann KH (1978) Phys Rev B 17:3757

6 Kvasnička V, Laurinc V and Biskupič S (1982) Phys Rep 90C: 160

7 Kümmel H, in Wu S S and Kuo T T S (eds) (1984) Nucleon-Nucleon Interaction and Nuclear Many-Body Problems: 46. World Scientific, Singapore

8 Bishop R F, in Wu S S and Kuo T T S (eds) (1984) Nucleon-Nucleon Interaction and Nuclear Many-Body Problems: 604. Horld Scientific, Singapore

9 Bishop R F and Kümet H (1987) Physics Today 40 (No 3): 52

10 Paldus J, Cižek $\mathrm{J}$ and Shavitt I (1972) Phys Rev A 5:50; Paldus J, Čižek J, Saute M and Laforgue A (1978) Phys Rev A 17:805

11 Lindgren I (1978) Int J Quantum Chem Symp 12:33

12 Bartlett R J and Purvis G D (1978) Int J Quantum Chem 14:561; Purvis G D and Bartlett R J (1982) J Chem Phys 76:1910; Laidig W D, Purvis G D and Bartlett R J (1985) J Phys Chem 89:2161; Sekino $H$ and Bartlett R J (1986) J Chem Phys 84:2726

13 Mukherjee D, Moitra R K and Mukhopadhyay (1975) Pramana (India) 4:247 and (1977) Mol Phys 33:955, Mukherjee D (1987) Int J Quantum Chem Symp 20:409, Lindgren I and Mukherjee D (1987) Phys Rep 151:93

14 Szalewicz K, Zabolitzky J G, Jeziorski B and Monkhorst H J (1984) J Chem Phys 81 : 2723

15 Bishop R F and Lührmann K H (1982) Phys Rev B 26:5523

16 Arponen J and Pajanne E (1982) J Phys C 15:2665, 2683

17 Emrich K and Zabolitzky J G (1984) Phys Rev B 30:2049

18 Hsue C S and Chern J L (1984) Phys Rev D 29:643;

Kaulfuss U B and Al tenbokum M (1986) Phys Rev D 33:3658;

Bishop R F and Flynn MF (1988) UMIST preprint

19 Hsue C S, Kümmel $H$ and Ueberholz P (1985) Phys. Rev. D 32:1435; Altenbokum M and Kümmel H (1985) Phys Rev D 32:2014;

Kaulfuss U (1985) Phys Rev D $32: 1421$; 
Hasberg G and Kümmel H (1986) Phys Rev C 33:1367;

Funke M, Kaulfuss U and Kümmel H (1987) Phys Rev D 35:621

20 Arponen J (1983) Ann Phys (NY) 151:311

21 Arponen J S, Bishop R F and Pajanne E (1987) Phys Rev A $36: 2519$

22 Arponen J S, Bishop R F and Pajanne E (1987) Phys Rev A 36:2539

23 Nesbet R K (1958) Phys Rev 109:1632

24 Hubbard J (1957) Proc Roy Soc London A240:539

25 Goldstone J (1957) Proc Roy Soc London A239:267

26 Brandow B H (1967) Rev Mod Phys $39: 771$ and (1970) Ann Phys (NY) 57:214

27 Emrich K (1981) Nucl Phys A351:379 and 397

28 Satake I (1975) Linear Algebra. Marcel Dekker, New York

29 Bohm D and Pines D (1951) Phys Rev 82:625 and (1953) 92:609

30 Blaizot J-P and Ripka G (1986) Quantum Theory of Finite Systems: 309. MIT Press, Cambridge, Mass.

31 Hohenberg $P$ and Kohn W (1964) Phys Rev 136:B864;

Kohn W and Sham L J (1965) Phys Rev 140:AT133 